\title{
Automated measurements of diffuse interstellar bands in early-type star spectra ${ }^{\star}$
}

\section{Correlations with the color excess}

\author{
L. Puspitarini, R. Lallement, and H.-C. Chen
}

\begin{abstract}
GEPI, Observatoire de Paris, CNRS, Université Paris Diderot, Place Jules Janssen, 92190 Meudon, France e-mail: [rosine.lallement; lucky.puspitarini; hui-chen.chen]@obspm.fr
\end{abstract}

Received 26 January 2013 / Accepted 4 April 2013

\section{ABSTRACT}

\begin{abstract}
Aims. Stellar spectroscopic surveys may bring useful statistical information on the links between diffuse interstellar bands (DIBs) and the interstellar environment. DIB databases can also be used as a complementary tool for locating interstellar (IS) clouds. Our goal is to develop fully automated methods of DIB measurements to be applied to extensive data from stellar surveys.

Methods. We present a method that is appropriate for early-type nearby stars, along with its application to high-resolution spectra of $\sim 130$ targets recorded with the ESO FEROS spectrograph and comparisons with other determinations. Using a DIB average profile deduced from the most reddened stars, we performed an automated fitting of a combination of a smooth stellar continuum, the DIB profile, and, when necessary, a synthetic telluric transmission.

Results. Measurements are presented for 16 DIBs in the optical domain that could be extracted automatically: 4726.8, 4762.6, 4963.9, 5780.4, 5797.1, 5849.8, 6089.8, 6196.0, 6203.0-6204.5, 6269.8, 6283.8, 6379.3, 6445.3, 6613.6, 6660.7, and 6699.3 A. Two approaches to determining equivalent width (fitted equivalent width and continuum-integrated equivalent width) were tested and the two determinations were found to agree within the uncertainties, except for the 6203-6204 multiple band, and are compatible with previous non automated measurements. Errors arising from statistical noise and continuum placement were estimated conservatively by means of the "sliding window" method. We derived the mean relationship between the DIB equivalent width and the color excess. Parameters of the linear correlation relationships are positively compared with published values, and most correlation coefficients were found to be higher than those based on an earlier type, UV bright target stars, which confirms previous results on the influence of the radiation field. We provide the first correlation parameters for the 5849.8, 6089.8, 6269.8, 6445.3, 6660.7, and $6699.3 \AA$ bands in the Galaxy. The coefficients are within the same range as for other optical bands.

Conclusions. Automated DIB equivalent width extractions can be performed for nearby early-type stars without significant losses in accuracy. This opens the perspective of building extended DIB catalogs based on high-resolution spectroscopic surveys. We discuss the limitations of this method in the case of distant stars and potential improvements.
\end{abstract}

Key words. ISM: lines and bands - dust, extinction

\section{Introduction}

Identifying the carriers of the diffuse interstellar bands (DIBs) is a longstanding challenge in astronomical spectroscopy. Large molecules or their ions appear as likely candidates, however identifications are still actively debated (see e.g. reviews by Herbig 1995; Sarre 2006; Friedman et al. 2011, and references therein). On the other hand, an increasing number of bands have been discovered in the optical domain (Jenniskens \& Desert 1994; Krelowski et al. 1995; Tuairisg et al. 2000; Weselak et al. 2010; Galazutdinov et al. 2000), with about 500 DIBs now identified between 4400 and $8600 \AA$ (Hobbs et al. 2008, 2009; McCall \& Griffin 2013).

As discussed by Friedman et al. (2011 and references therein), attempts to identify DIBs can be divided into four fields: (1) searches for signatures in the laboratory; (2) theoretical modeling of the detected profiles; (3) searches for correlations of DIBs with other interstellar parameters; and (4) searches

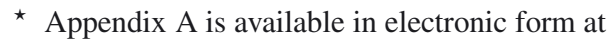
http: //www . aanda.org for correlations between individual pairs of DIBs. Extensive datasets, in particular from stellar spectroscopic surveys, can be useful in the last two perspectives. However, analyses of large data sets require automated methods of DIB extraction. As part of this work we built and tested such methods.

Another advantage of extensive DIB measurements from spectroscopic surveys is their potential use as a tracer of the IS matter to build three-dimensional (3D) maps, by means of the derived spatial gradients of their strengths. This is made possible by the link between the DIBs and the amounts of intervening IS matter. Such a link has been extensively studied for extinction (or color excess) and atomic or molecular tracers. Many of them are significantly or weakly correlated with interstellar integrated quantities, namely the $\mathrm{HI}, \mathrm{H}_{2}$ column or the color excess, as shown by numerous studies (e.g. Friedman et al. 2011; Vos et al. 2011). In particular, the correlation of the DIB strength with the extinction or the color excess has been investigated for a large number of bands, and it has been shown that the degree of correlation is highly variable among the DIBs (see e.g. Moutou et al. 1999 and references in Table 1). For some of them (e.g. Friedman et al. 2011; Ordaz et al. 2011), the correlation 
is moderate to good, and sometimes improved when restricted to regions that are not exposed by a strong UV radiation field (Raimond et al. 2012).

As a matter of fact, there is a strong and complex influence of the UV radiation field, which has now been studied in detail (e.g. Krelowski \& Sneden 1994; Salama et al. 1996; Sonnentrucker et al. 1997; Cordiner et al. 2008; Vos et al. 2011). In particular, two distinct types of sightlines with respect to DIB properties and UV extinction law have been identified (Krelowski \& Sneden 1994; Krelowski et al. 1995): the $\zeta$-type sightlines correspond to UV-shielded cloud cores, while the $\sigma$-type sightlines probe external regions of the clouds, which are partially ionized by UV radiation field. The DIBs behave differently in response to those conditions, and there are families of DIBs that respond in a similar way (e.g. Krelowski \& Walker 1987). Even if their degree of correlation with the dust or gas column is poor, DIBs can be used to locate IS clouds in the same way as interstellar lines are used, simply by detecting gradients that mark the cloud boundary; e.g., neutral sodium is very poorly correlated with HI. Nevertheless, abrupt jumps in neutral sodium columns clearly mark the boundary of atomic or partially ionized clouds.

DIBs would be complementary to other tracers that have been used for IS mapping, such as atomic lines or color excesses (Welsh \& Lallement 2010; Vergely et al. 2010). The easily observed IS lines, such as the NaI D1-D2 and the CaII H-K lines, saturate for moderate and high reddening so that the uncertainties associated to the derived gas columns are large. This is a problem when one uses spatial gradients of the absorbing columns to locate the IS clouds. At variance with those lines, most of the DIBS in the visible do not suffer from these saturation effects and can potentially be used for longer distances. With the advent of spectroscopic surveys, such as the ESOGaia Spectroscopic Survey (Gilmore et al. 2012), large numbers of DIB absorption measurements are expected, which can feed databases for 3D mapping. Thanks to the Gaia mission, parallax distances should become available for billions of stars and allow 3D interstellar matter (ISM) maps to be built by means of inversion methods (Vergely et al. 2001, 2010). Even though assigning distances to clouds by means of absorption data does not require any proportionality between the measured quantity (column of a gaseous species, reddening, or DIB strength) and the column of IS gas or dust, the existence of an established average relationship is useful for estimating average volume opacities of 3D dust clouds, in addition to their locations. Because the better the DIB correlation is, the more meaningful the estimation of the dust (or gas) column that will be based on the DIB strength, it is interesting to study all correlation coefficients in detail. In another respect, the possibility of estimating the extinction based on DIB strength and the correlation law may be extremely valuable in case photometric or spectrophotometric data are absent or ambiguous. As part of the present work, we extend previous correlative studies and provide new average relationships for several DIBs.

In a first paper (Raimond et al. 2012), we extracted two of the strongest and well studied interstellar bands ( $\lambda \lambda$ 5780.4, 6283.8) from about 150 high-quality, high-resolution early-type spectra of nearby stars and performed a study of their correlations with the color excess. We found overall agreement with previous studies, in particular the work of Friedman et al. (2011). This comparison showed that the dispersion around the average relationship is diminished when using later type target stars, and it was argued that this is due to the decrease in radiation field influences. In fact, a short sightline may be dominated by dense clouds located immediately in front of the target stars and strongly irradiated.

In this previous study, the measurement of the DIB equivalent width (EW) was performed in a classical, non automated way similar to the methods used by several other groups, i.e., by means of a preliminary fit of the continuum around the DIB to a polynomial, a subsequent normalization of the spectrum, and computation of the absorption area below the normalized continuum. At variance with this earlier work, we here performed automated DIB measurements, and we compared their results with those from the previous interactive method. As said above, automated methods are mandatory in the case of spectroscopic surveys. Here we have been testing methods based on template DIB profiles, in the same way Cordiner et al. (2008) proceeded in the case of M31.

We have developed an automated program and make use here of the same database to test it and extract a series of measurable DIBs, namely the $\lambda \lambda$ 4726.83, 4762.61, 4963.88, 5780.38, $5797.06, \quad 5849.81,6089.85,6195.98,6203.05-6204.49$, $6269.85,6283.84,6379.32,6445.28,6613.62,6660.71$, and 6699.32 DIBs. The central wavelengths here are the values listed in the recent catalog of Hobbs et al. (2008). An exception is the $6204 \AA$ complex, which has been shown to be made of two distinct absorptions centered on 6203.05 and $6204.49 \AA$ and corresponding to two distinct carriers (see e.g. Porceddu et al. 1991). For simplicity here we have chosen to derive the equivalent width of the ensemble of those two absorptions, with an approximate centering on $6204 \AA$. For the two strong DIBs of our first paper, we compare the derived equivalent widths with our previous non automated analysis results. We finally compare the DIB strengths with the color-excess values, and the derived relationships with previously established results, when they exist.

Section 2 describes the data, while Sect. 3 presents the spectral analysis and the error estimates. Section 4 presents the results, and describes the relationships with the color excess, as well as comparisons with previous works. Section 5 discusses the limitations of the automated method and potential improvements.

\section{Data}

We use high-resolution $(R \approx 48000)$, high signal-to-noise $(S / N \geq 100)$ spectra of nearby, early-type (B to A5), fastrotating stars that are mostly located within $400 \mathrm{pc}$. Observations were done with the FEROS spectrograph at the ESO/Max Planck $2.2 \mathrm{~m}$ telescope in La Silla and were part of the LP179.C-0197 program. FEROS provides a broad spectral range: $\sim 3530$ to $\sim 9210 \AA$. This dataset has been previously used for interstellar (IS) neutral sodium and ionized calcium measurements that, after merging to an existing database, have been inverted to produce 3D maps of the nearby IS matter (Welsh \& Lallement 2010; Vergely et al. 2010). They have also been used to study the two strong bands at 5780.38 and $6283.84 \AA$ (Raimond et al. 2012). Such high-quality and wide-range spectra are well suited for the study of diffuse bands, including weak ones.

For the purpose of comparisons with the reddening, we use homogeneous dataset of color-excess data based on the seven-color Geneva photometric system (Cramer 1999; Burki \& Cramer, priv. comm.). Those data, which have also been used by Raimond et al. (2012), were all determined based on the same calibration method. The homogeneity avoids additional dispersion and biases in the correlation computation. We thus restricted 
this study to target stars possessing a color excess determination from this single source. This reduced the number of useful targets from about 400 to 150 . The spectra were also preliminarily analyzed in the search for narrow lines due to cold companions. As a matter of fact, narrow and weak stellar features may contaminate the DIBs and make the continuum fitting difficult or biased. This resulted in excluding a significant fraction of binary stars. The final number of targets available for the fully automated DIB extraction is 129 .

The Geneva $E(B-V)$ color excess values were converted to Strömgen $E(b-y)$ color excesses, using all Strömgen data compiled by Vergely et al. (2010) for which both determinations do exist. A scaling factor was derived from the cross-correlation and a similar cross-correlation was then performed to convert to the commonly used Johnson system. The relationship with the Johnson system is $E(B-V)($ Geneva $)=1.703 E(B-V)$ (Johnson). Finally, the error on $E(B-V)$ was estimated conservatively to be 0.03 , as discussed in Raimond et al. (2012).

\section{Data analysis}

\subsection{Choice of DIBs}

We started with the recent and extended DIB catalog of Hobbs et al. (2008) and initially considered all potential bands. DIBs were first selected when they were strong enough to be detected in more than about 20 target stars, in such a way that a correlation with the color excess can be established with some statistical significance. Because our targets are nearby and slightly reddened, this resulted in a drastic selection of the strongest bands, despite the high $\mathrm{S} / \mathrm{N}$. In a second step, we excluded the strong but very broad and shallow bands such as the $4426 \AA$ band. In the case of our early-type stars, those bands have widths that become comparable to the stellar line widths and are difficult to disentangle from those stellar lines in an automated way (those DIBs are paradoxically easier to measure for late-type stars). For the selection we used a threshold of $\gtrsim 0.05$ for the ratio between the DIB equivalent width and the full width at half maximum (FWHM). For those broad and shallow bands, methods based on stellar spectral models (Chen et al. 2013) would be more appropriate and will be the subject of future work. Table 1 shows our 16 selected DIBs and lists their parameters as determined by Hobbs et al. (2008). They have all been detected in several objects, including in the Magellanic clouds, M31, M33, and for nine of them previous determinations of the relationship with the reddening do exist for Milky Way targets and are listed in the table.

\subsection{Fitting method}

Our goal is to measure DIB equivalent widths (EWs) in series of stellar spectra in an entirely automated way. There are several steps in this process. First, prior to the actual spectral analysis, we establish an empirical DIB shape model. Alternatively, we use a pre-existing model from prior studies. This is the case for the 6283.84 DIB profile, which is taken from Raimond et al. (2012) and is already adapted to the resolution and instrumental response. When weak telluric lines are present, we use a telluric transmission model that is fitted in airmass and Doppler shift to the data and is used to eliminate those features. Then, we fit a pre-defined spectral interval around the DIB to a combination of a smooth continuum, represented by a polynomial function, here of third to seventh-degree, and the DIB model. The DIB model is allowed to vary in strength and velocity shift, and the polynomial
Table 1. List of the selected DIBs, their parameters, and relevant studies.

\begin{tabular}{ccccl}
\hline \hline $\begin{array}{c}\lambda_{\mathrm{c}}^{*} \\
(\AA)\end{array}$ & $\begin{array}{c}F W H M \\
(\AA)\end{array}$ & $\begin{array}{c}E W \\
(\mathrm{~m} \AA)\end{array}$ & $\begin{array}{c}\Delta E W \\
\text { rms err. }\end{array}$ & Ref. \\
\hline 4726.83 & 2.74 & 283.8 & 4.2 & 4,13 \\
4762.61 & 1.00 & 50.8 & 1.6 & $4,7,13$ \\
4963.88 & 0.62 & 53.4 & 1.0 & $4,7,13$ \\
5780.38 & 2.11 & 257.0 & 3.0 & $1,2,3,4,6,7,8,9^{n G}, 10,11^{n G}$, \\
& & & & $12^{n G}, 14,15,18$ \\
5797.06 & 0.77 & 199.0 & 1.1 & $1,2,3,4,5,6,7,8,9^{n G}, 10,11^{n G}$, \\
& & & & $12^{n G}, 14,17,18$ \\
5849.81 & 0.82 & 95.6 & 1.2 & 1,4 \\
6089.85 & 0.54 & 28.0 & 0.8 & 4 \\
6195.98 & 0.42 & 37.8 & 0.6 & $2,4,14,16,17,18,19$ \\
$6203.05 ;$ & $1.21 ;$ & $57.1 ;$ & $1.7 ;$ & $2,4,7,9^{n G}, 11^{n G}, 12^{n G}, 14,16,17$ \\
6204.49 & 4.87 & 71.6 & 6.9 & \\
6269.85 & 1.17 & 77.0 & 1.7 & $2,4,7,11^{n G}, 12^{n G}$ \\
6283.84 & 4.77 & 459.7 & 6.9 & $2,4,7,9^{n G}, 11^{n G}, 12^{n G}, 14,15,16,17$ \\
6379.32 & 0.58 & 94.9 & 0.8 & $4,5,7,11^{n G}, 12^{n G}, 18,19$ \\
6445.28 & 0.46 & 35.7 & 0.7 & 4 \\
6613.62 & 0.93 & 165.1 & 1.4 & $4,5,7,8,9^{n G}, 11^{n G}, 12^{n G}, 14,17,18$ \\
6660.71 & 0.58 & 33.0 & 0.9 & $4,11^{n G}$ \\
6699.32 & 0.63 & 21.6 & 0.9 & 4,17 \\
\hline
\end{tabular}

Notes. ${ }^{(*)} \lambda_{\mathrm{c}}$, FWHM, EW, and $\Delta \mathrm{EW}$ from Hobbs et al. (2008) (measurements towards HD 204827). ${ }^{(n G)}$ extragalactic target: M31, M33, etc.

References. (1) Josafatsson \& Snow (1987), reflection nebulae targets; (2) Benvenuti \& Porceddu (1989), link to the 2175 A feature; (3) Herbig (1993), correlations with gaseous lines and extinction; (4) Jenniskens \& Desert (1994), DIBs survey with $E W / E(B-V)$; (5) Sonnentrucker et al. (1997), Ionization properties; (6) Krełowski et al. (1999), relation with $\mathrm{CH}$ and $\mathrm{CH}+$; (7) Thorburn et al. (2003), relation with $C_{2}$ molecules; (8) Destree et al. (2007), cool stars; (9) Cordiner et al. (2008), M31; (10) van Loon et al. (2009), Tarantula survey; (11) Cordiner et al. (2011a), M31; (12) Cordiner et al. (2011b), M31, M33; (13) Ordaz et al. (2011), O stars; (14) Friedman et al. (2011), Correlations with gaseous lines sand color excess; (15) Raimond et al. (2012), survey: correlation with color excess; (16) Chen et al. (2013), cool giant bulge stars; (17) Xiang et al. (2012), compilation of correlations; (18) Vos et al. (2011), study of environmental effects; (19) Walker et al. (2001), study of profiles.

coefficients are all free to vary. The spectral interval over which the fit is done is pre defined, and it contains the DIB and broad enough intervals both short ward and long ward of the DIB to ensure a good fit of the stellar continuum. When strong telluric lines are present, instead of the combination of a DIB model and a smooth continuum, we fit the data to a triple combination of telluric transmission model (also fit in strength and shift), DIB model, and smooth continuum. In all cases (non existent, weak, or strong telluric lines), by using such a global fitting, we avoid any manual selection of intervals for the continuum fit.

The DIB EW is derived in two separate ways. The first way assumes that the continuum is reasonably fitted during the fit and also that the actual DIB absorption has a shape that is similar to the model. In this case the EW is simply the product of the model EW by the fit coefficient (or DIB's strength or power index of the absorption), since for our sightlines the linear regime prevails. The second estimate is solely based on the first, less restrictive assumption that the continuum has been correctly fitted during the DIB+continuum(+telluric) global fit, but does NOT make use of the DIB model or of the fit coefficient. It simply uses the area between the spectrum and the normalized continuum. Following Cordiner et al. (2008), we call the two measurements as the fitted EW (based on the DIB model and power index) and continuum-integrated EW (independent of the DIB model). 
The comparison between the two EW values allows potential discrepancies between the observed and modeled DIB shapes to be detected, including in particular some DIB broadening due to a velocity shift spread of the interstellar clouds, or DIB shape variations with sightline. As a matter of fact, using a unique DIB shape is equivalent to assuming that sub structures in the DIB profile vary in the same proportions from one sightline to the next. This is only true at first order, and it is known from high-resolution spectral analyses that the shape may vary in some cases (e.g., Galazutdinov et al. 2008). It is also equivalent to assuming that the DIB is much broader than the velocity shift between the intervening clouds. This is in principle justified when the DIBs are relatively broad, and especially broad in comparison with the radial velocity dispersion of the IS clouds. Conversely, if for a target the two estimates of the EW are in good agreement, this implies that the actual DIB shape in the stellar spectrum is very similar to the template shape. This also implies that the continuum has been realistically fitted and the DIB EW well estimated, and constitutes a validity check of the automated method. We will come back to this point in the last section.

The DIB model was obtained empirically during the preliminary phase in the following way. We selected a small number (about 3 to 5) of targets, such as HD 172488, HD 147932, HD 176853, HD 151884, and HD 171957, that have high color excess values and excellent spectra, extracted the DIB in a classic way and scaled them to the same EW. Then, we simply averaged them and used the mean as a template. In the case of the weakest DIBs or the $6204 \AA$ blend, the model has to be used carefully. As we mentioned before, the comparison of the two measurements, the fitted and continuum-integrated EWs, will allow its reliability to be checked.

Atmospheric lines were modeled by means of a synthetic telluric transmission computed using the line-by-line radiative transfer model (LBLRTM) code and the HIgh-ResolutionTRANsmission molecular absorption (HITRAN) spectroscopic database, here for a standard atmosphere profile and for the altitude of La Silla (Clough et al. 2005; Rothman et al. 2009). For the 6283.8 DIB, telluric lines of diatomic oxygen are particularly strong. For this DIB, and this DIB only, the correction was not done in a preliminary phase, but instead we fitted the data to a triple combination of stellar, DIB, and telluric models, as described above. During the fit, both the DIB and the telluric transmission are free to vary in Doppler shift and strength, allowing for Earth velocity and airmass variations from one spectrum to the other. An illustration of the fitting can be found in Fig. 1. For the 5849.8, 6269.85, 6445.28, 6613.62, and 6660.71 DIBs that are only slightly contaminated, we more simply used the synthetic telluric transmission to perform a correction prior to the DIB measurement (see Fig. 2). Finally, in a few cases we also applied the classical corrections before the automated fit to correct for cosmic rays, by means of a classical interpolation in the contaminated area. We also applied masks in the case of neighboring DIBs to separate their respective absorptions (e.g. for the 6195.98 DIB). For some of the cooler targets, we also applied masks to avoid the contamination by narrow stellar lines as described in detail in Raimond et al. (2012).

The consecutive steps of the EW measurement method can be found in Fig. 3, and the illustration of the whole fitting process for the 6195.98 DIB can be found in Fig. 4. For the equivalent width measurements, we used the same limits of integration (DIB broadness) as Friedman et al. (2011) for 5780.38, 6204, 6283.84, 6195.98, 6613.62, and 5797.06 A. Other DIB shapes, and integration limits can be seen in Figs. 6 .

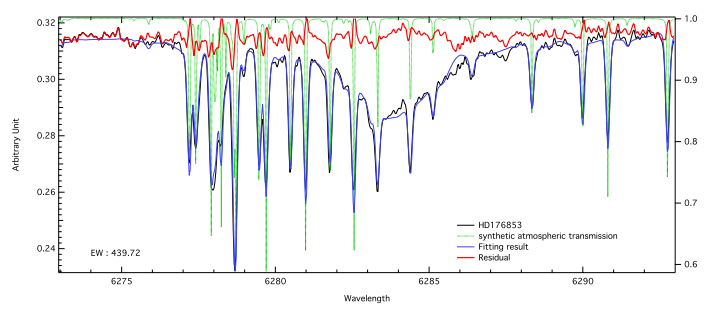

Fig. 1. Illustration of telluric line corrections using an automated fitting method that takes stellar continuum, telluric lines, and also DIB into account. The black line is the original spectrum of HD 176853, the green line is the synthetic telluric transmission, the blue line is the fitting result, and the red line is the residual. The very strong telluric lines are not entirely removed, but the residuals do not significantly affect on the DIB equivalent width.

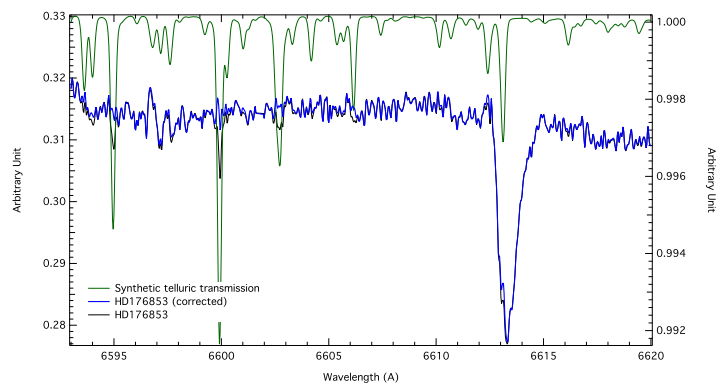

Fig. 2. Example of a preliminarily correction for weak telluric lines. The black line is the spectrum of HD 176853, the green line is the synthetic telluric transmission, and the blue line is the corrected spectrum.

\subsection{Error estimates and results}

\subsubsection{Error estimates}

Errors on the equivalent width are of two kinds: first, the statistical noise in the spectrum, pixel-to-pixel, and second, the error due to uncertainties in defining the continuum. The latter corresponds to the departure between the extrapolated part of the fitted continuum and the true underlying continuum at the DIB location. By definition it is impossible to know its exact shape, and those departures depend on the quality of the spectrum and of the properties of the target (mainly temperature and intrinsic variability). We estimated this potential departure in an empirical, statistical way by means of the sliding windows method described in detail by Raimond et al. (2012). In short, we applied the same continuum fitting, based on two spectral intervals of the same width and separated by the same gap, but this time by centering the gap NOT at the DIB location but at a large number of locations in the spectrum, in regions on both sides of the DIB that are free of IS absorption. To do so, we translated (or slid) the fitting intervals along the spectrum in a quasi-continuous way (hence the sliding window name). The difference between the fitted continuum (which corresponds to the extrapolated part that would be used if there was a DIB) and the actual spectrum provides an estimate of the error during the continuum placement. The entire set of locations provides a list of error values from which we can extract a standard deviation. We then quadratically added this value to the error due to the measurement uncertainties $\operatorname{err}_{\text {noise }}=\sigma \times \frac{\Delta \lambda}{N}$, where $\sigma$ is a standard deviation, $\Delta \lambda$ the interval of DIB absorption, and $N$ number of points in the interval of absorption. 


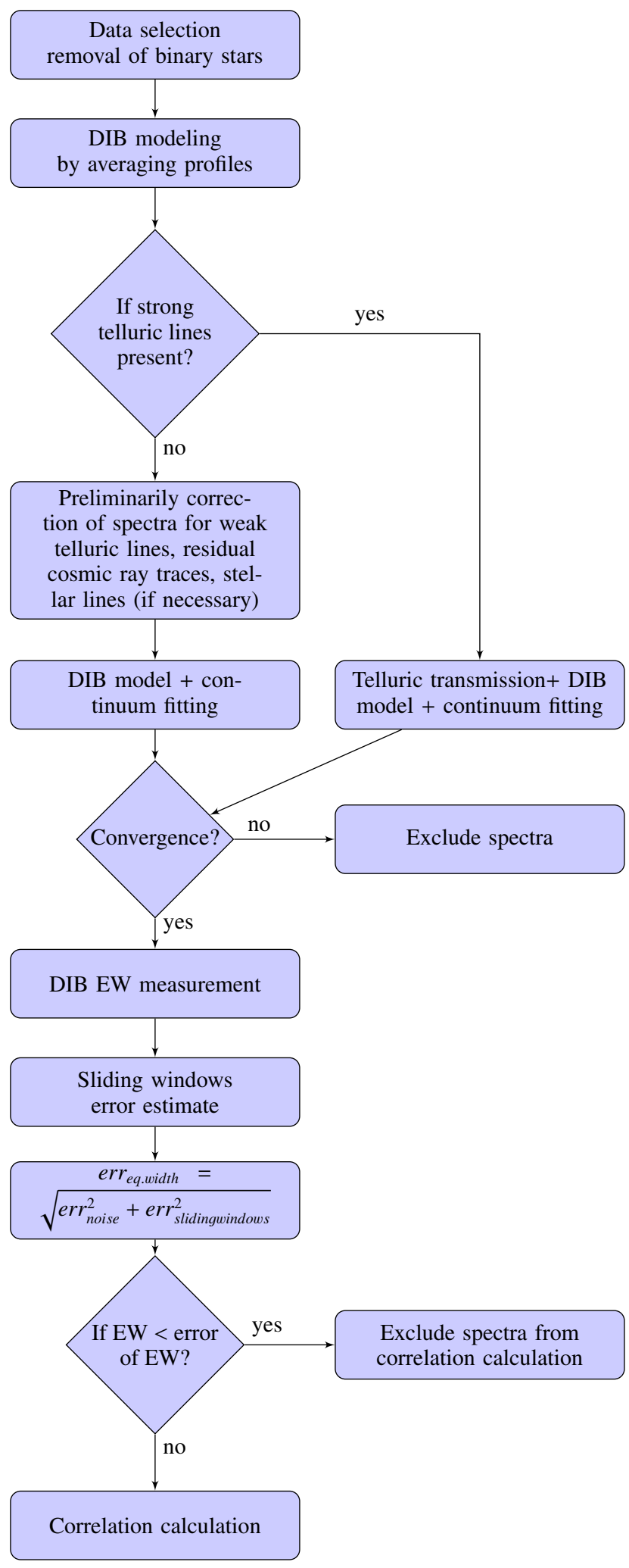

Fig. 3. DIB fitting process.

\subsubsection{Results and comparison between the two determinations of the equivalent width}

Once the automated DIB fitting is performed, it is possible to compute the equivalent width in two ways, as detailed in Sect. 3.2: the first is based on the result of the continuum + DIB template fitting and is a simple scaling of the DIB template
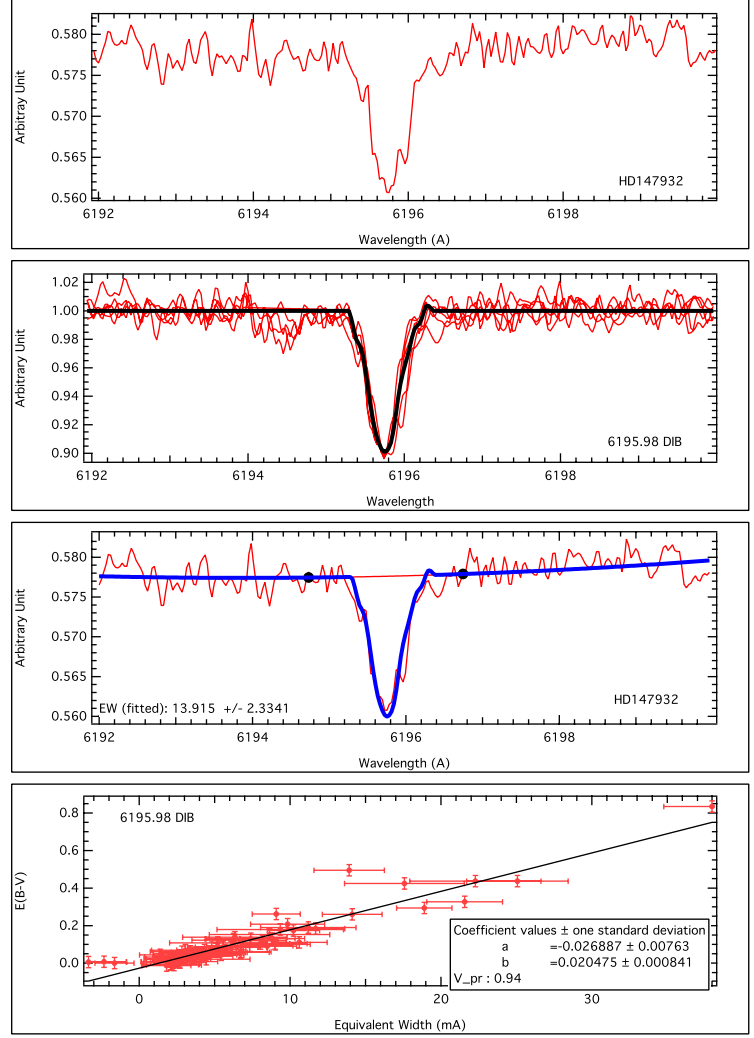

Fig. 4. Illustration of the automated DIB fitting method for the 6196.0 DIB. The first row image shows the spectrum of HD 147932 (red line) with the DIB band at about $6195.98 \AA$. The second row image shows an empirical DIB shape model (black line). It is an average profile deduced from profiles of the most reddened stars, scaled to the same EW. The third row image shows an example of automated DIB fitting (DIB model + continuum fitting) The red line is the spectrum of target star and the blue line is the fitting result. The last row shows the correlation between EWs and color excess values using OLS method.

EW. The second consists in measuring the absorption area directly in the normalized spectrum, based on the fitted continuum. Differences between the two arise if the DIB shape in the spectrum differs significantly from the template, and as said above their similarity is a validity test of the assumptions and of the automated method. We have thus carefully compared the two equivalent widths for the various DIBs.

Figure 5 shows their comparison for the 16 DIBs. It can be seen that for almost all DIBs the differences are inferior to the uncertainties in the very large majority of measurements. As said above, DIB shape variabilities and velocity spread of the interstellar clouds must be reflected in the differences between the two EWs. The absence of significant differences may be explained here by the proximity of the target stars, which results in a small velocity dispersion. It also implies that the DIB shape is representative enough of the majority of the absorptions in the nearby ISM. For that reason, we can use any of the EWs to study the correlations with other parameters. The exception is the 6203-6204 $\AA$ DIB, and this is certainly related to the fact that there are two components and that their relative strengths do vary from one target to the other. For this system it may be interesting to consider both the fitted EW and the continuumintegrated $\mathrm{EW}$ in the search for correlations (see the two results in Table 2). 

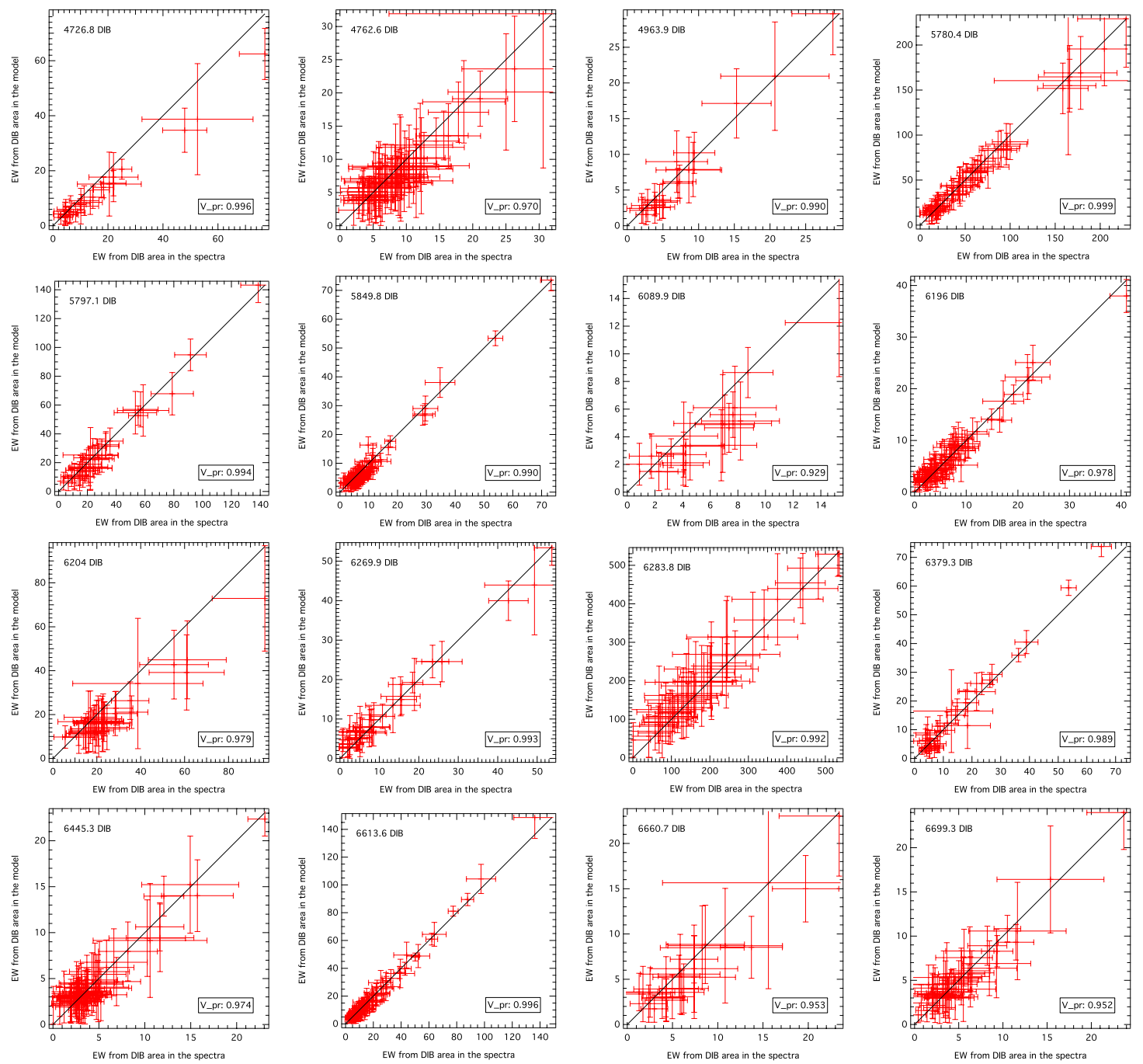

Fig. 5. Comparison of results from the two methods of EW measurements, the fitted EWs vs. the continuum-integrated EWs.

Table A. 1 contains the list of target stars, their coordinates, the color excess as determined from the Geneva photometry (see Sect. 4), and the measured equivalent width (the fitted EWs). The results show that the DIB absorptions are weak and that we are far from saturation, as expected for our nearby targets.

\section{Correlation with the color excess}

We used the Geneva determinations of $E(B-V)$ (Cramer 1999; Burki \& Cramer, priv. comm.) to compute the average linear relationships of the sixteen DIBs with the color excess. This was done in two ways:

- by performing a simple linear fitting without taking individual errors into account on both quantities (ordinary least square, OLS).

- by applying the orthogonal distance regression (ODR) method (or total least-squares method), taking individual errors on both the EWs and the $E(B-V) \mathrm{s}$ into account. For this method, we used the ODRPACK95 package (Boggs et al. 1989) implemented in the IGOR 6.22A WaveMetrics software.

Following most of the studies, we did not impose a strict proportionality between the two quantities. We performed the correlation exclusively for the target stars and EWs listed Table A.1, i.e., excluding binary stars or stars for which the automated fit did not converge owing to the combination of noise level and DIB weakness, and also stars for which the measured EW is smaller than the estimated uncertainty. The Pearson correlation coefficient is deduced from the OLS method, with two values listed, the first one for the totality of the measurements, and the second after removal of two peculiar stars, when they have not been already removed from the list due to the criteria mentioned above.

The correlation parameters and coefficients can be found in Table 2, and the linear fits are displayed in Figs. 6. We provide the first correlation parameters and coefficients for six DIBs, namely the $5849.81,6089.85,6269.85,6445.28,6660.71$, and $6699.32 \AA$ band. It is interesting to note that the new correlations coefficients are in the same range as for the other DIBs in the optical, with Pearson coefficients between 0.89 and 0.95 , which confirms a significant positive correlation between color excess and EW of those DIBs. Since the Pearson coefficient does not take measurement uncertainties into account, it is also informative to consider the uncertainties on the slopes obtained from the OLS and ODR methods. In the case of the OLS method, uncertainties on the parameters are estimated along with the fitting process from the dispersion around the mean value. In the ODR method, the uncertainties are calculated based on all measurement uncertainties. 

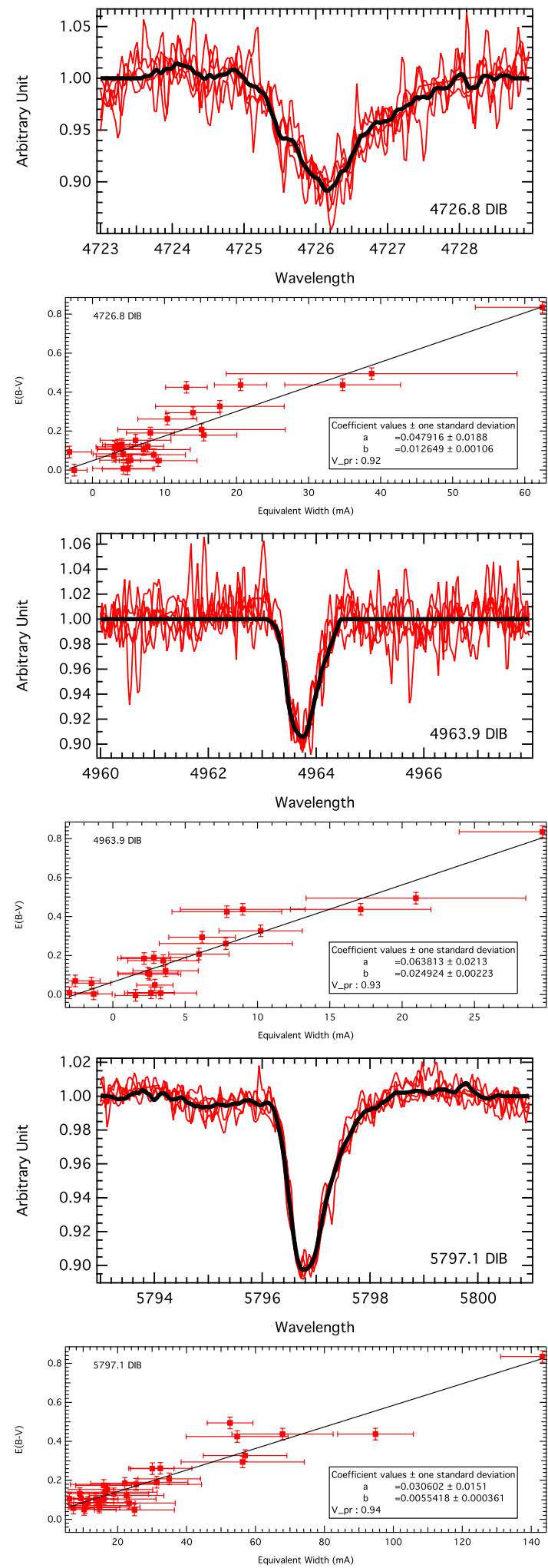

Fig. 6. DIB model and color excess-DIB equivalent width correlation.

We compared our results with the relevant published values from references listed in Table 1. Correlation studies of the 4726.8, 4762.6, and 4963.9 ^ DIBs have been performed by Ordaz et al. (2011) for a large set of galactic $\mathrm{O}$ stars. While our correlation coefficient is similar for DIB 4726.8 (0.92 vs. 0.90), it is significantly higher for the two other DIBs. Following the same argument as in Raimond et al. (2012), we believe this is due
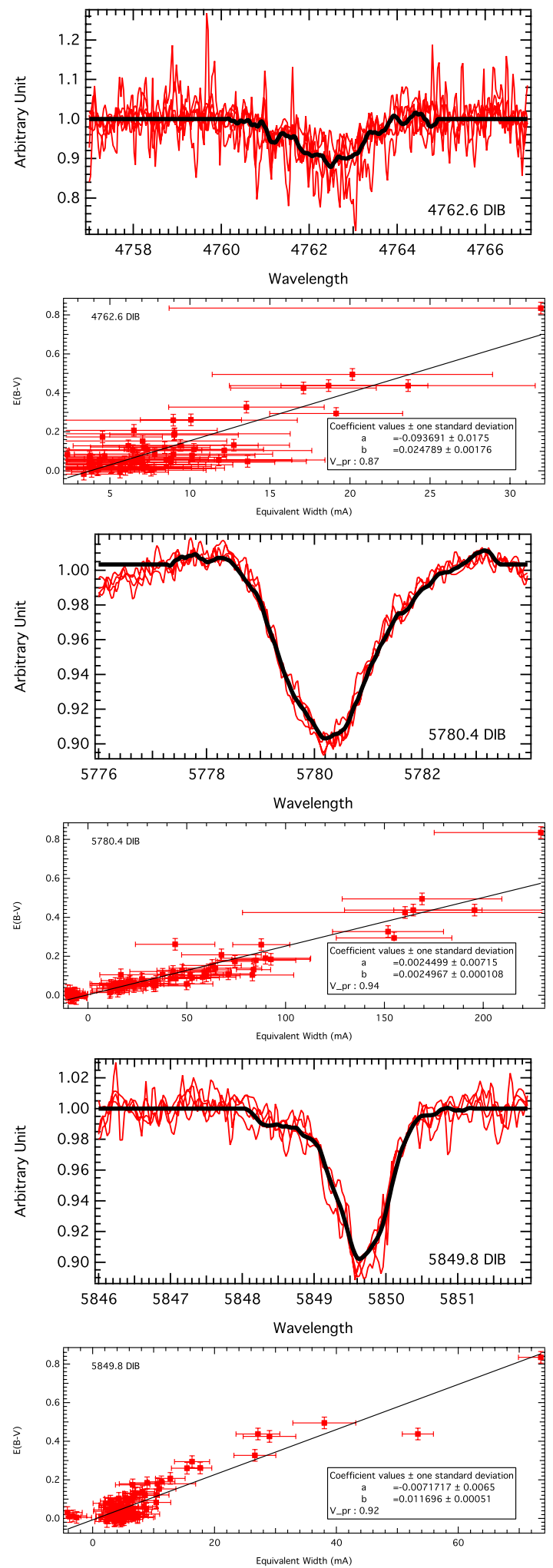

to the characteristics of our targets, which are late B or A stars. Their relatively low UV radiation field partly avoids strong effects on the DIB carriers in clouds that are located close to the targets, while such effects must be present in a number of their less distant $\mathrm{O}$ stars. This suggests that the 4726.8 DIB is less sensitive to such effects. Friedman et al. (2011) have established correlations with the color excess for six DIBs in common with 

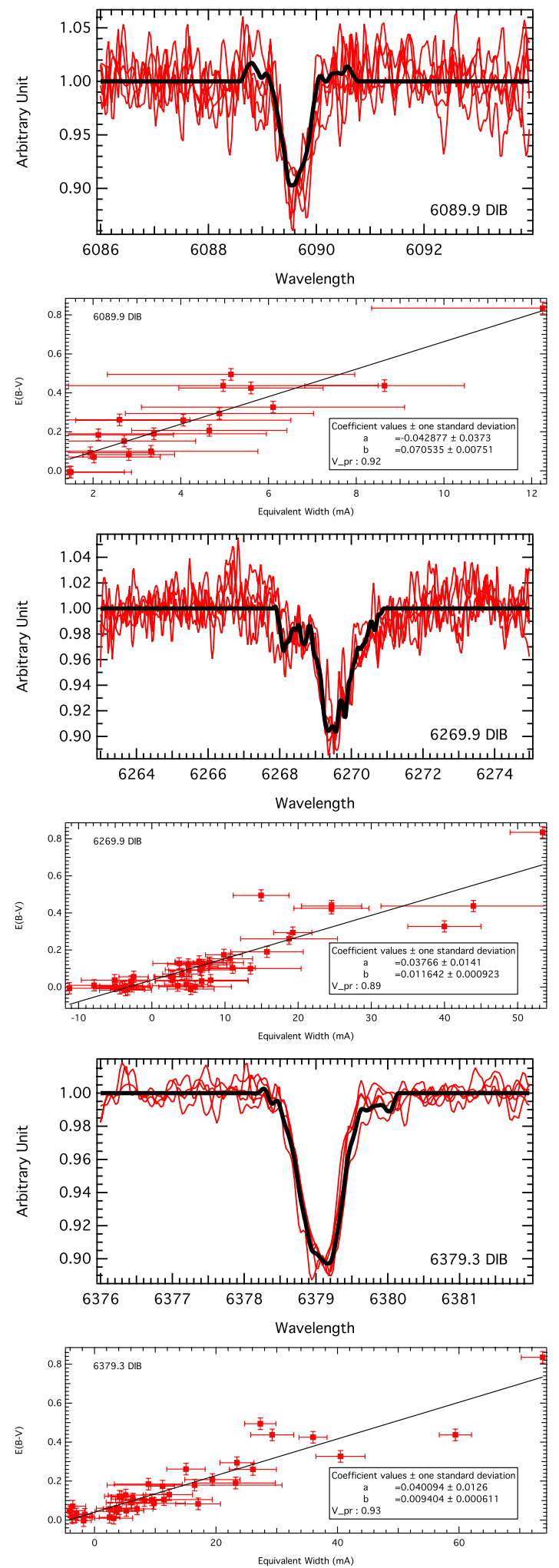

Fig. 6. continued.

our study, namely the 5780.4, 5797.1, 6196.0, 6204, 6283.8, and 6613.6 DIBs, using O-and early B stars from the northern hemisphere, while Raimond et al. (2012) have established correlations for the 5780.4 and 6283.8 DIBs using the same set of cooler stars as we are using here. In general, our results agree with theirs, as well as with earlier measurements from Herbig (1993). Figure 7 shows the comparison between our results and
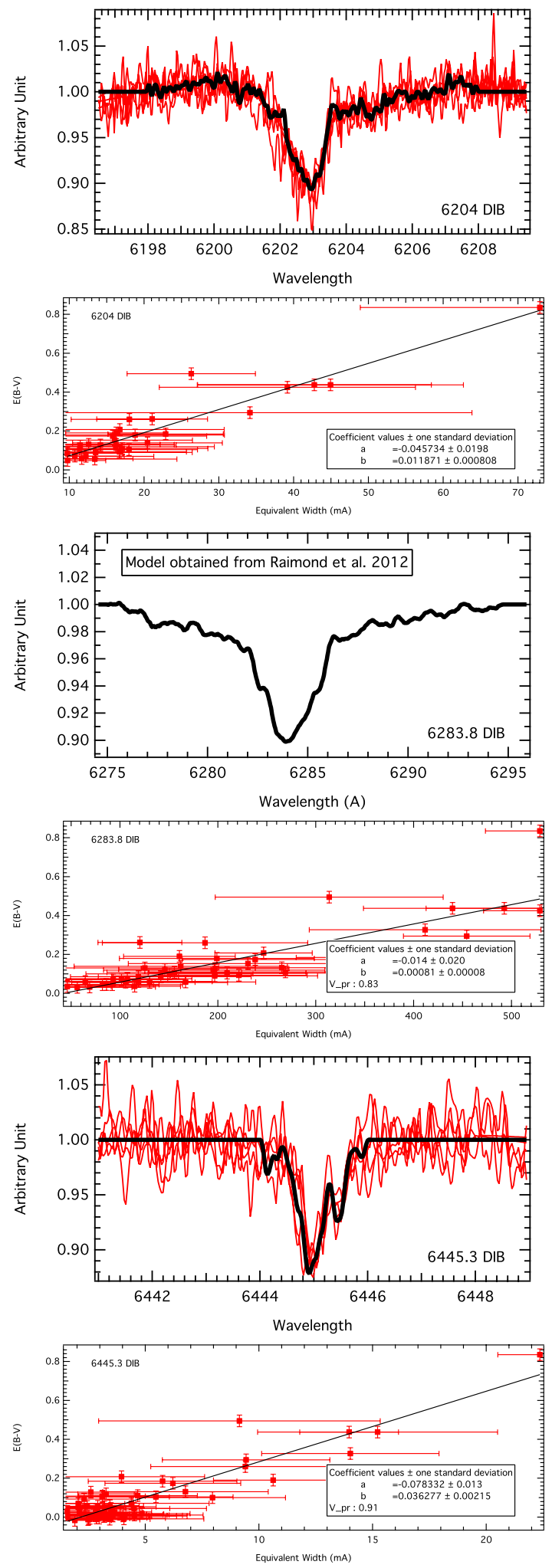

Raimond et al. (2012). There are only small differences with Raimond et al. (2012) for the DIB 5780.4, which are probably caused by the noise and residual contamination in the spectra during the fitting process. We find a noticeable difference in the case of the 6283.8 (a slope of 0.00076 instead of 0.00067 $\mathrm{mag} / \mathrm{mA}$ ), which we explain by the following bias. For this DIB, the automated sliding window method is influenced by telluric 

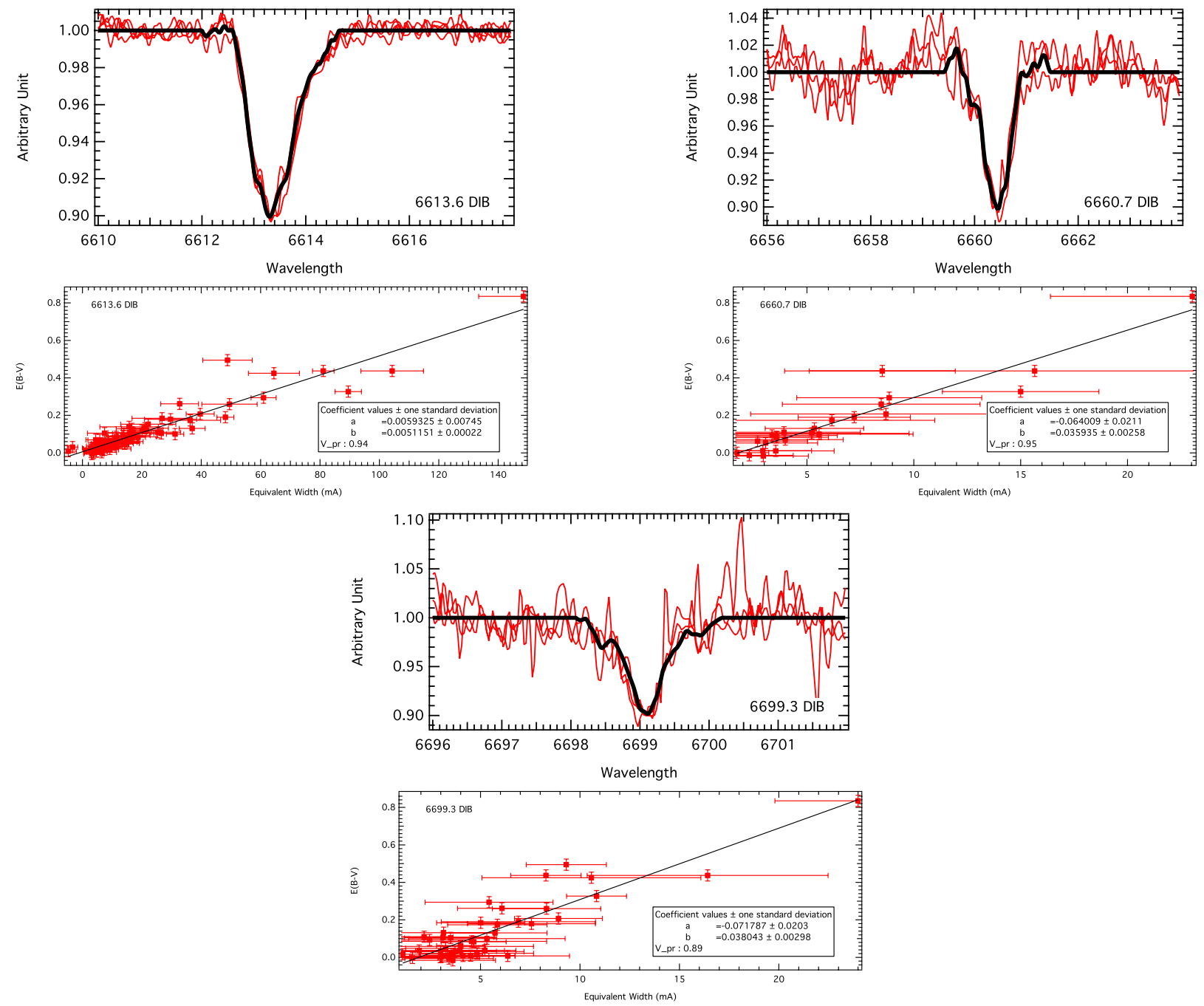

Fig. 6. continued.
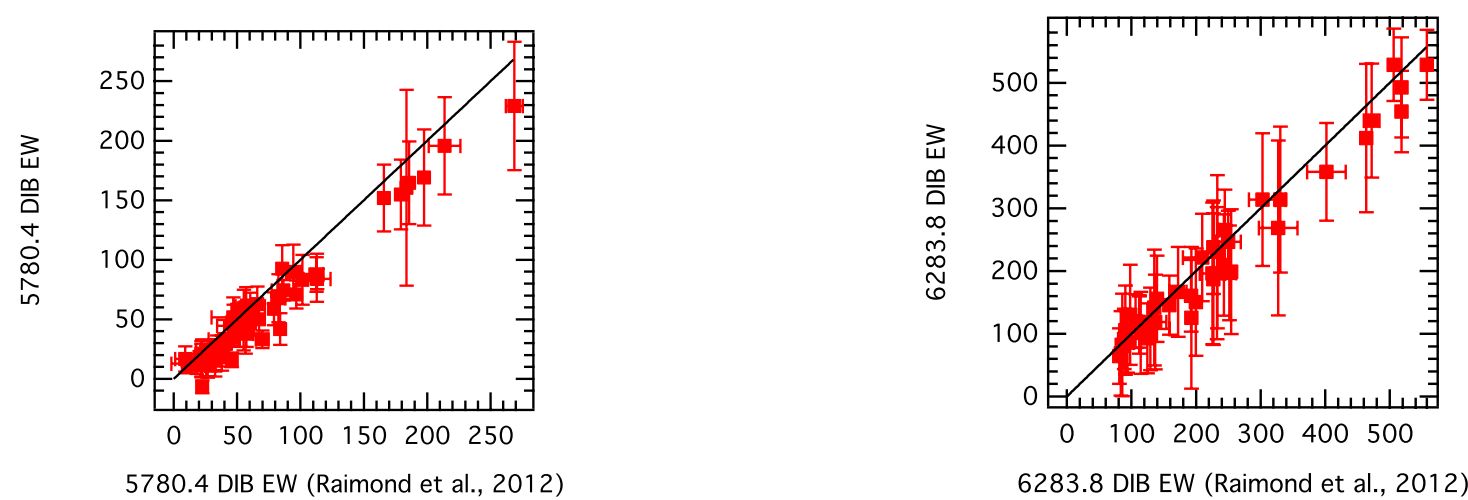

Fig. 7. Comparison of equivalent width value of 5780.38 DIB (left) and 6283.84 DIB (right) with Raimond et al. (2012).

lines and gives very large uncertainties on EWs, which result in the exclusion of a large number of targets with small EWs, following our threshold rule. This introduces a bias towards higher EWs for low reddening values and a larger slope. This implies that such a method is not fully appropriate where telluric lines are too strong and that another method should be sought. Similarly to the case of the $5780.4 \AA \mathrm{DIB}$, for which using cooler stars results in a tighter correlation, we note here that for the 5797.1, 6196.0, 6204, and 6613.6 DIBs, we also obtain a significantly better correlation than the one of Friedman et al. (2011). This is very likely also caused by environment effects, since those bands are strongly influenced by the radiation field. The same remarks apply to the correlation coefficients measured by Vos et al. (2011) for the upper Scorpius region that, except for DIB 5797, are lower than found here. Josafatsson \& Snow (1987) computed Pearson coefficients for three DIBS in common, 5780, 5849, and $5797 \AA$, using early-type target stars in reflection nebulae. We also computed their correlation 


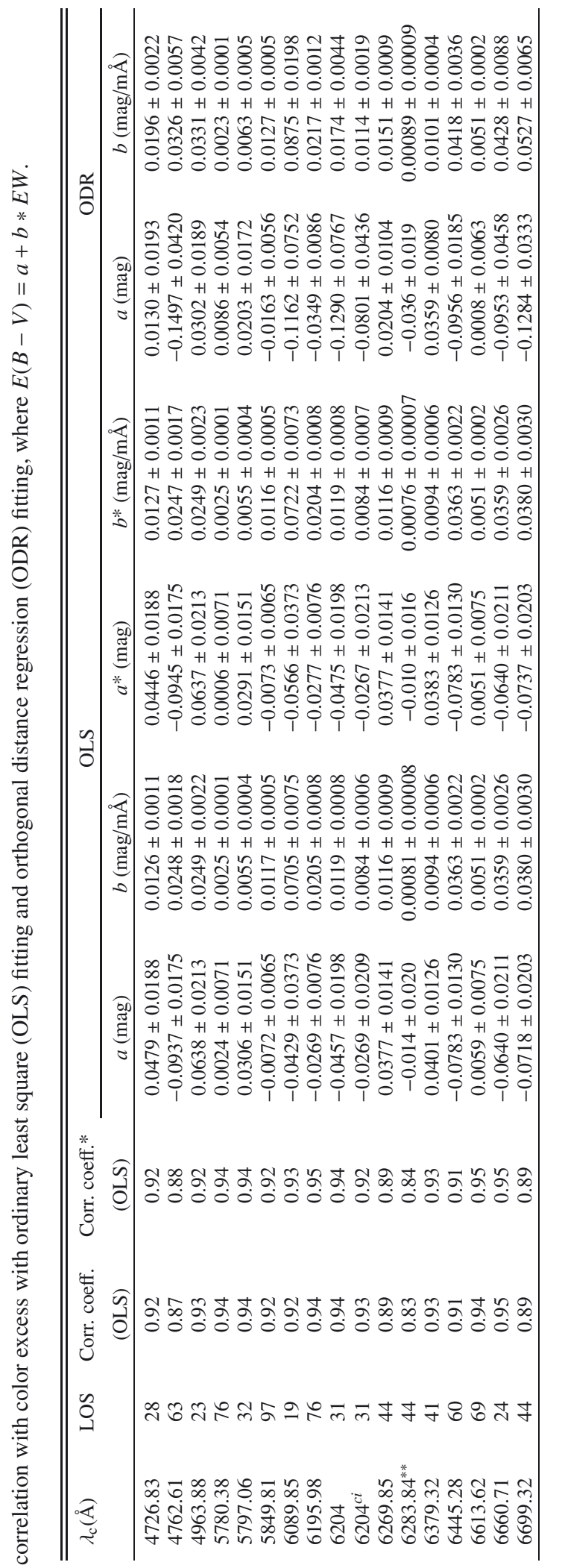

苚

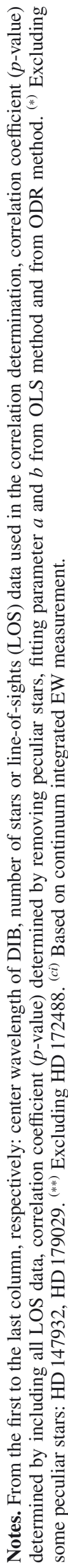

parameters, using data from their tables. The parameters agree with ours, within the uncertainties. Our Pearson coefficients are higher: 0.94 vs. 0.90 for DIB 5780, 0.94 vs. 0.89 for DIB 5797, and finally 0.92 vs. 0.75 for DIB 5849. The large difference for this last band suggests that it is very sensitive to radiation conditions. Finally, Cordiner et al. (2011a) quotes average coefficients of 88 and $50 \mathrm{~mA} / \mathrm{mag}$ for the 6379 and 6660 DIBs, respectively, in their comparison with M31. Those values are similar to our results.

\section{Discussion}

We have devised and applied a simplified but automated method of DIB equivalent width measurements appropriate to early-type spectra. The method uses a preliminary DIB shape and fits the data to the product of a smooth, polynomial continuum and a DIB template profile fitted in strength and velocity shift. The scaling coefficient, combined with the EW of the template of reference, provides a first determination of the DIB equivalent width (the fitted EW). A second estimate of the equivalent width is the absorption directly measured below the normalized spectrum using the fitted continuum, independently of the DIB template (the continuum-integrated EW). At variance with the first, this second determination takes the departures from the initial shape into account, the first step being a way to obtain a realistic continuum at the DIB location.

We applied this method to a database of nearby stars highresolution spectra, for stars possessing a precise determination of the color excess. We preliminarily eliminated binary systems and corrected the spectra for weak telluric lines by means of a modeled atmospheric transmission. In the case of the strong telluric lines that contaminate the $6283.8 \AA \mathrm{DIB}$, we also tested the fit of the triple combination of a telluric transmission, a smooth continuum and a DIB template. We estimated the uncertainties on the DIB EWs due to the noise level and continuum placement.

For all DIBs except one, we found that the two EW determinations are in good agreement, and are compatible within our estimated uncertainties. This implies that, at least for our database, there are only small variations of the DIB profiles from the initial templates. The main reasons are the small number of absorbing clouds and the small radial velocity differences among those clouds, in comparison with the DIB intrinsic width. For more distant targets, departures between the two EWs may be used to initiate a second fit with two or more DIB profiles centered at distinct velocity shifts, in an iterative manner. The exception to the equality of the two determinations is the 6203-6204 DIB, which is composed of at least two distinct bands (see e.g. Porceddu et al. 1991).

Correlations parameters with the stellar reddening are found to be in good agreement with previous determinations, again within uncertainties, and we present six new determinations. The Pearson correlation coefficients are between 0.89 for 6269.85 and $6699.32 \AA$ to 0.95 for $6660.71 \AA$, in a very similar range to other DIBs in the optical domain. When compared with previous determinations, our Pearson coefficients for all DIBs are often significantly higher. We believe that this is due to the lack of strong stellar radiation field influences, because our targets are cooler than most of those used in other studies (e.g. Friedman et al. 2011; Ordaz et al. 2011).

We note that the two DIBs that correlate the best with the reddening, the 6196 and $6614 \AA$ bands, are also those that have high degrees of correlation with each other and other bands, such 


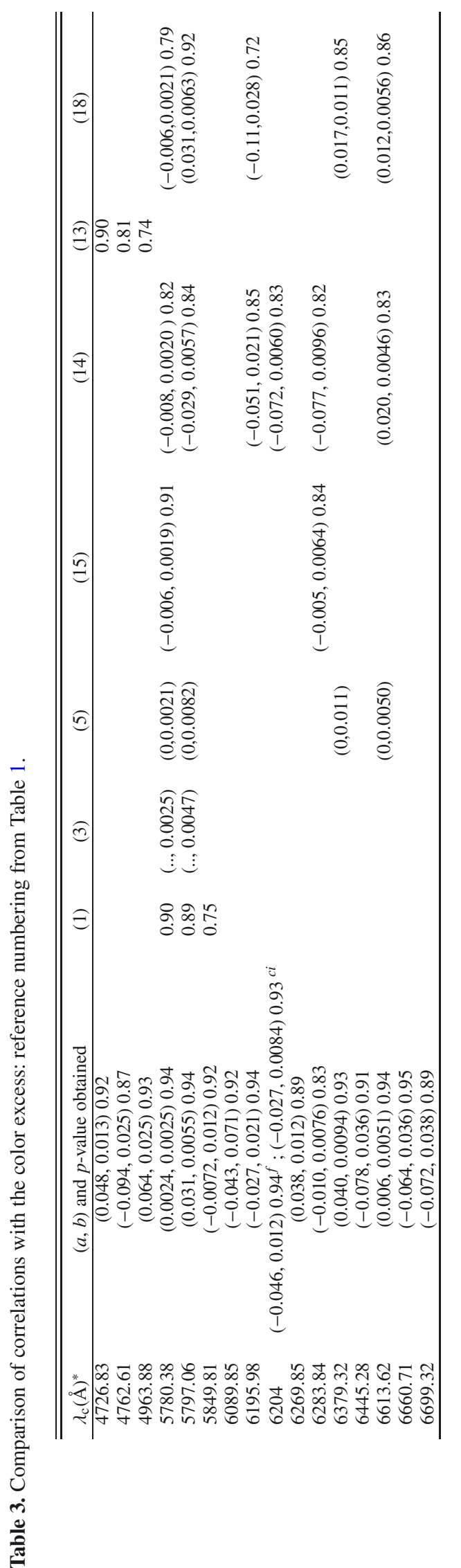

as 5544, 5809, $6699 \AA$ (see details on correlations in the recent work by Xiang et al. 2012 and McCall et al. 2010).

We conclude from this study that DIBs have been correctly extracted from spectroscopic data of early-type stars in the simplified automated way we tested. Such treatments may be applied to building extended databases, available for correlative studies, and studies of the environmental effects. The forthcoming ESA cornerstone Gaia mission will provide the distances to most targets observable with Galactic spectroscopic surveys, opening the way to three-dimensional mapping of the DIB carriers based on absorption measurements from surveys. In this perspective, the development of refined automated methods is mandatory. A condition of success for such methods is the elimination of binary stars, which must be detected prior to the DIB extraction. Here our targets are relatively nearby, and the sightline IS velocity dispersion is small in comparison with the DIB width. However, for distant targets, procedures allowing for multiple clouds with radial velocity shifts potentially broadly distributed will be necessary. They could be, e.g., based on iterations for an increasing number of components, using differences between fitted DIB EWs and continuum-integrated EWs as criteria for the number of clouds, following the above remarks on those differences. On the other hand, if one wants to reach the best possible spatial resolution of the 3D maps, methods appropriate for cool stars will be required.

Such methods are currently in development, and promising results have been obtained from combinations of stellar synthetic spectra of cool stars, telluric transmission profiles, and DIB absorption templates (Chen et al. 2013). Again, special attention will have to be given to the case of binary systems, for which automated tools may provide erroneous results. A preliminary detection of those systems will have to precede the DIB analysis itself.

Acknowledgements. We thank the referee for the useful comments on the manuscript. Part of this work has been funded by the French Research Agency in the frame of the STILISM project. H.-C. C. wants to acknowledge support by grants from Région Ile-de-France.

\section{References}

Benvenuti, P., \& Porceddu, I. 1989, A\&A, 223, 329

Boggs, P. T., Byrd, R. H., Donaldson, J. R., \& Schnabel, R. B. 1989, ODRPACK, ACM Trans. Mathematical Software, 15, 348

Chen, H.-C., Lallement, R., Babusiaux, C., et al. 2013, A\&A, 550, A62

Clough, S. A., Shephard, M. W., Mlawer, E. J., et al. 2005, J. Quant. Spectrosc. Radiat. Transf., 91, 233

Cordiner, M. A., Cox, N. L. J., Trundle, C., et al. 2008, A\&A, 480, L13

Cordiner, M. A., Cox, N. L. J., Evans, C. J., et al. 2011a, ApJ, 726, 39

Cordiner, M., Cox, N. L. J., Smith, K. T., \& Evans, C. J. 2011b, BAAS, 43, \#112.04

Cramer, N., 1999, New Astron. Rev., 43, 343

Destree, J. D., Snow, T. P., \& Eriksson, K. 2007, ApJ, 664, 909

Friedman, S. D., York, D. G., McCall, B. J., et al. 2011, ApJ, 727, 33

Galazutdinov, G. A., Musaev, F. A., Krełowski, J., \& Walker, G. A. H. 2000 PASP, 112, 648

Galazutdinov, G. A., Manicò, G., Pirronello, V., \& Krełowski, J. 2004, MNRAS, 355,169

Galazutdinov, G. A., LoCurto, G., \& Krełowski, J. 2008, ApJ, 682, 1076

Gilmore, G., Randich, S., Asplund, M., et al. 2012, The Messenger, 147, 25

Herbig, G. H. 1993, ApJ, 407, 142

Herbig, G. H. 1995, ARA\&A, 33, 19

Hobbs, L. M., York, D. G., Snow, T. P., et al. 2008, ApJ, 680, 1256

Hobbs, L. M., York, D. G., Thorburn, J. A., et al. 2009, ApJ, 705, 32

Jenniskens, P., \& Desert, F.-X. 1994, A\&AS, 106, 39

Josafatsson, K., \& Snow, T. P. 1987, ApJ, 319, 436

Krelowski, J., \& Sneden, C. 1994, The First Symposium on the Infrared Cirrus and Diffuse Interstellar Clouds, 58, 12

Krelowski, J., \& Walker, G. A. H. 1987, ApJ, 312, 860

Krelowski, J., Sneden, C., \& Hiltgen, D. 1995, Planet. Space Sci., 43, 1195 
Krełowski, J., Ehrenfreund, P., Foing, B. H, et al. 1999, A\&A, 347, 235

McCall, B. J, \& Griffin, R.E. 2013, Proc. R. Soc. A, 469,2151

McCall, B. J., Drosback, M. M., Thorburn, J. A., et al. 2010, ApJ, 708, 1628

Moutou, C., Krełowski, J., D’Hendecourt, L., \& Jamroszczak, J. 1999, A\&A, 351,680

Ordaz, M. P., Appellániz, J. M., Ballano, A. S., et al. 2011, Highlights of Spanish Astrophysics VI, eds. M. R. Zapatero Osorio, J. Gorgas, J. Maíz Apellániz, J. R. Pardo, \& A. Gil de Paz., 544

Porceddu, I., Benvenuti, P., \& Krelowski, J. 1991, A\&A, 248, 188

Raimond, S., Lallement, R., Vergely, J.-L., Babusiaux, C., \& Eyer, L. 2012, A\&A, 544, A136

Rothman, L. S., Gordon, I. E., Barbe, A., et al. 2009, J. Quant. Spec. Radiat. Transf., 110, 533

Salama, F., Bakes, E. L. O., Allamandola, L. J., \& Tielens, A. G. G. M. 1996, ApJ, 458, 621

Sarre, P. J. 2006, J. Mol. Spectrosc., 238, 1

Sonnentrucker, P., Cami, J., Ehrenfreund, P., \& Foing, B. H. 1997, A\&A, 327, 1215
Tarantola, A., \& Valette, B. 1982a, J. Geophys., 50, 159

Thorburn, J. A., Hobbs, L. M., McCall, B. J., et al. 2003, ApJ, 584, 339

Tuairisg, S. O., Cami, J., Foing, B. H., Sonnentrucker, P., \& Ehrenfreund, P. 2000, A\&AS, 142, 225

van Loon, J. T., Smith, K. T., McDonald, I., et al. 2009, MNRAS, 399, 195

Vergely, J.-L., Freire Ferrero, R., Siebert, A., \& Valette, B. 2001, A\&A, 366 , 1016

Vergely, J.-L., Valette, B., Lallement, R., \& Raimond, S. 2010, A\&A, 518, A31

Vos, D. A. I., Cox, N. L. J., Kaper, L., Spaans, M., \& Ehrenfreund, P. 2011, A\&A, 533, A129

Walker, G. A. H., Webster, A. S., Bohlender, D. A., \& Krełowski, J. 2001, ApJ, 561,272

Welsh, B. Y., \& Lallement, R. 2010, PASP, 122, 1320

Welsh, B. Y., Lallement, R., Vergely, J.-L., \& Raimond, S. 2010, A\&A, 510, A54

Weselak, T., Galazutdinov, G. A., Han, I., \& Krełowski, J. 2010, MNRAS, 401, 1308

Xiang, F., Liu, Z., \& Yang, X. 2012, PASJ, 64, 31 
L. Puspitarini et al.: Automated DIB measurements

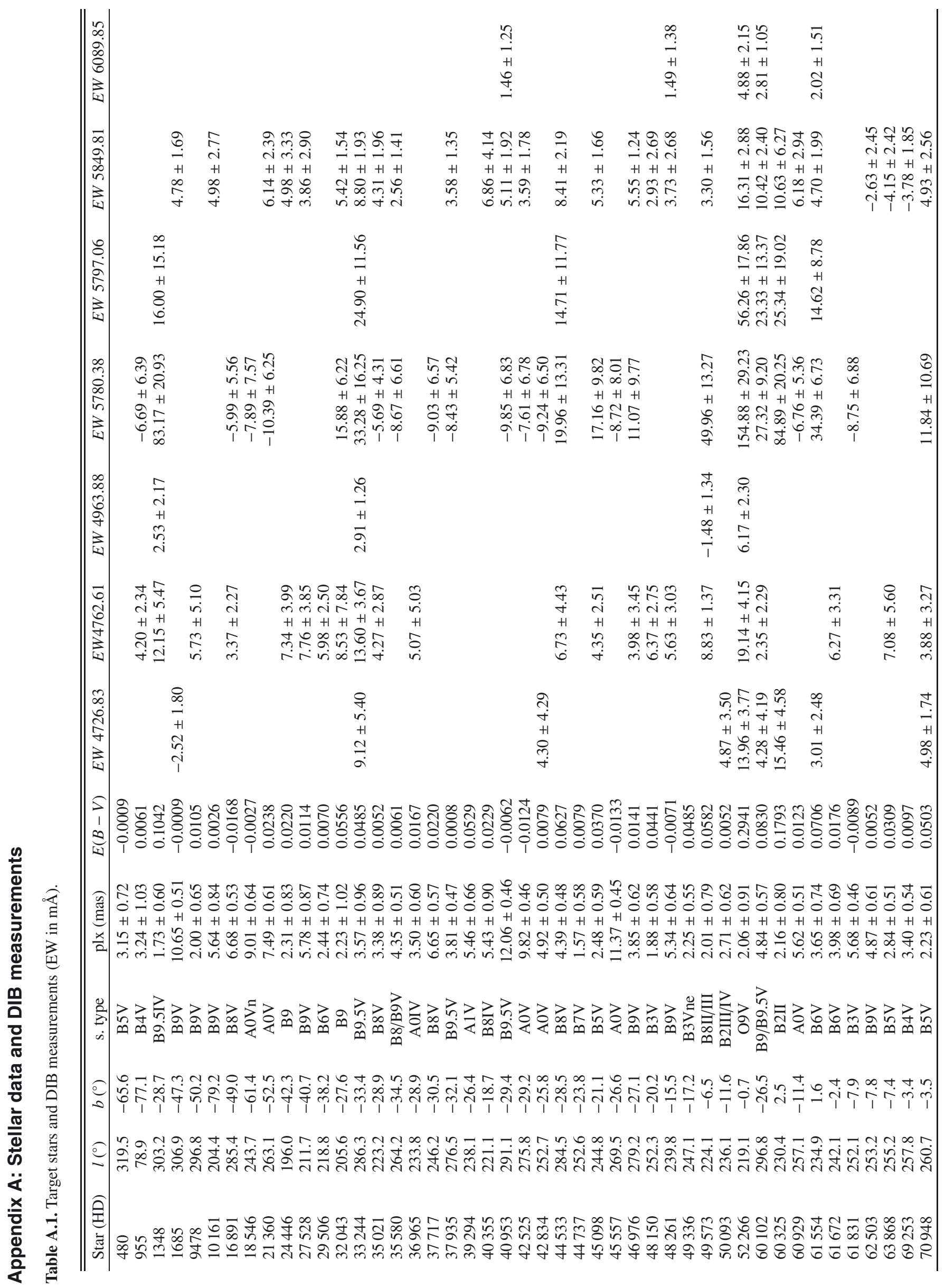




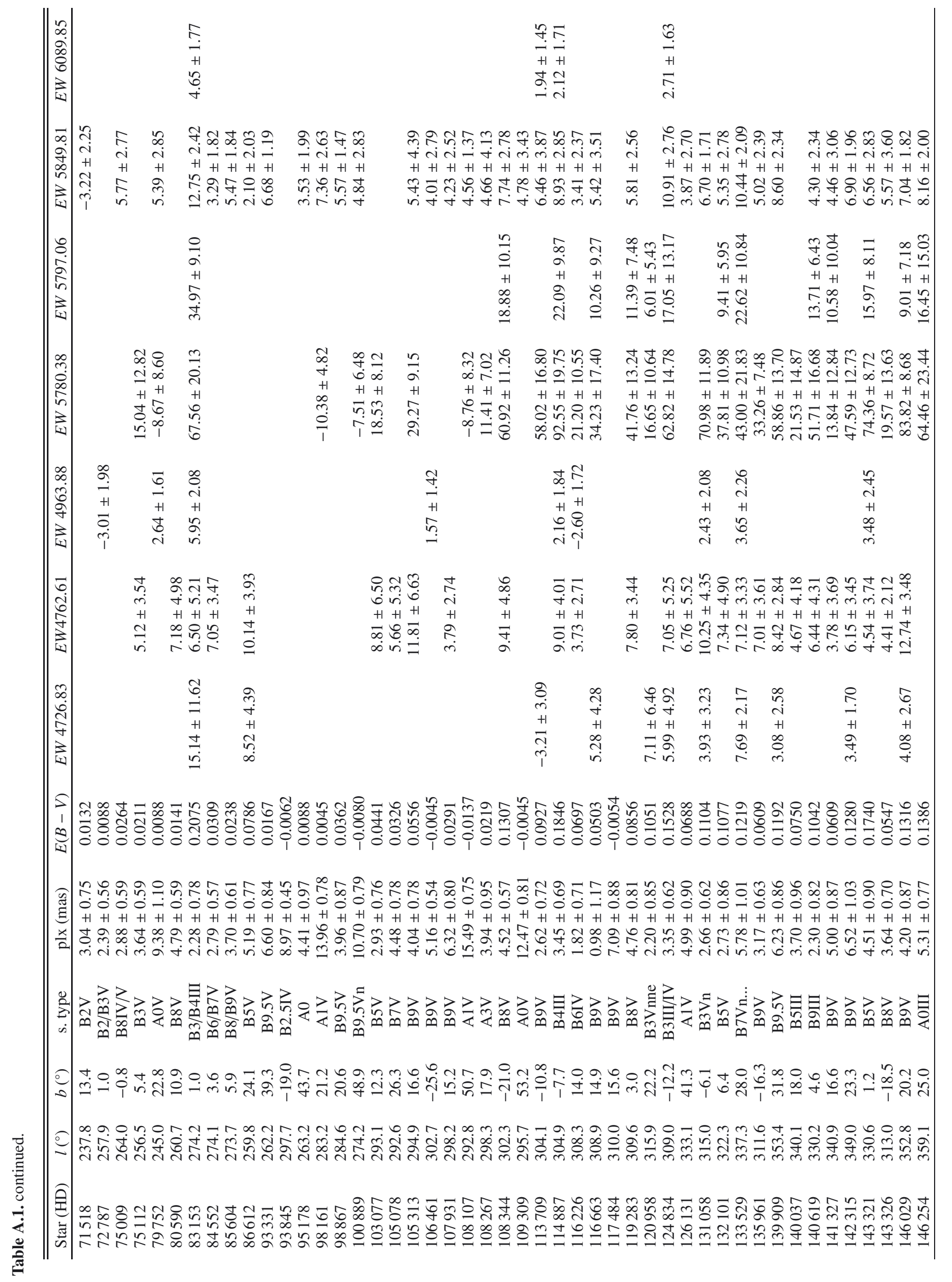




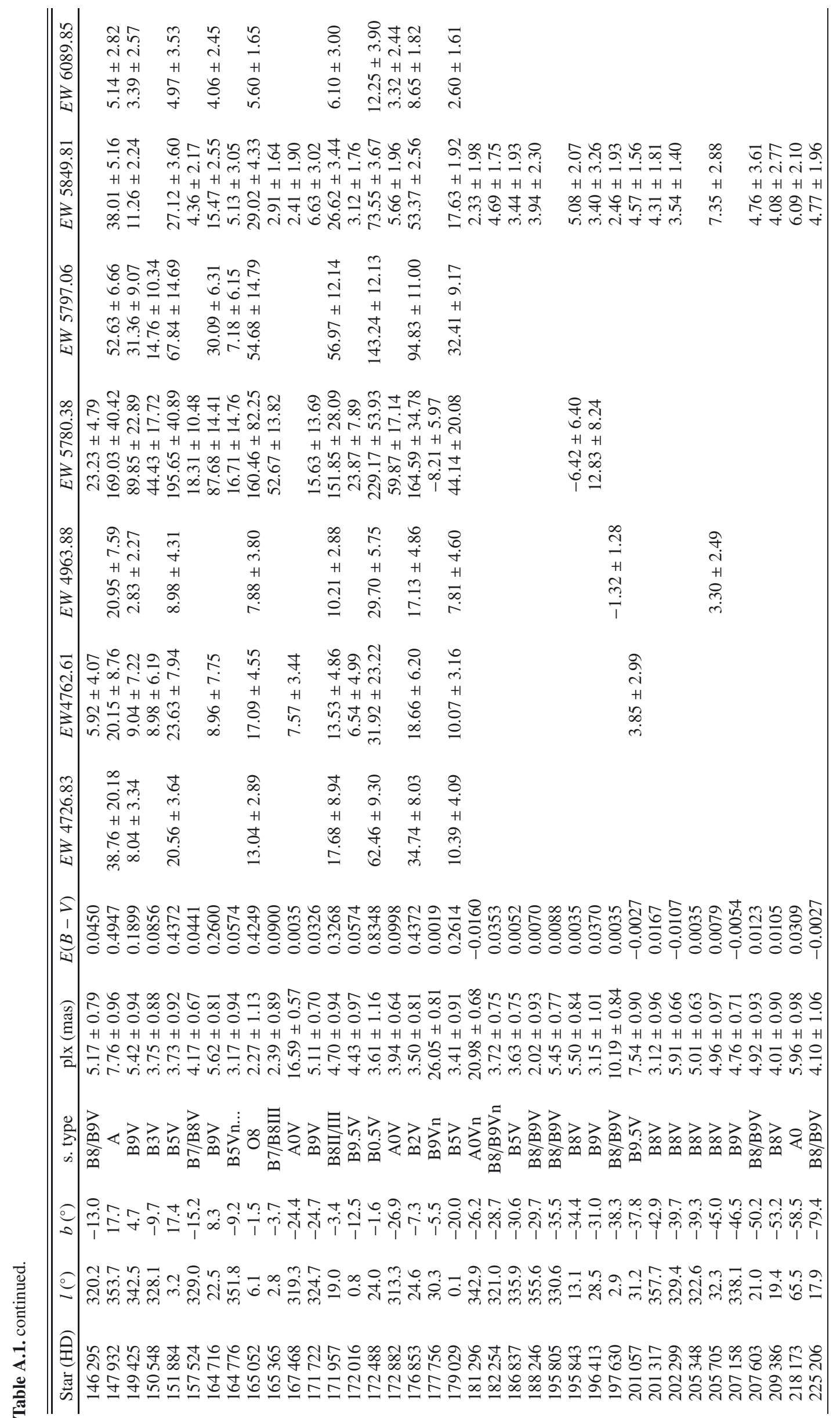




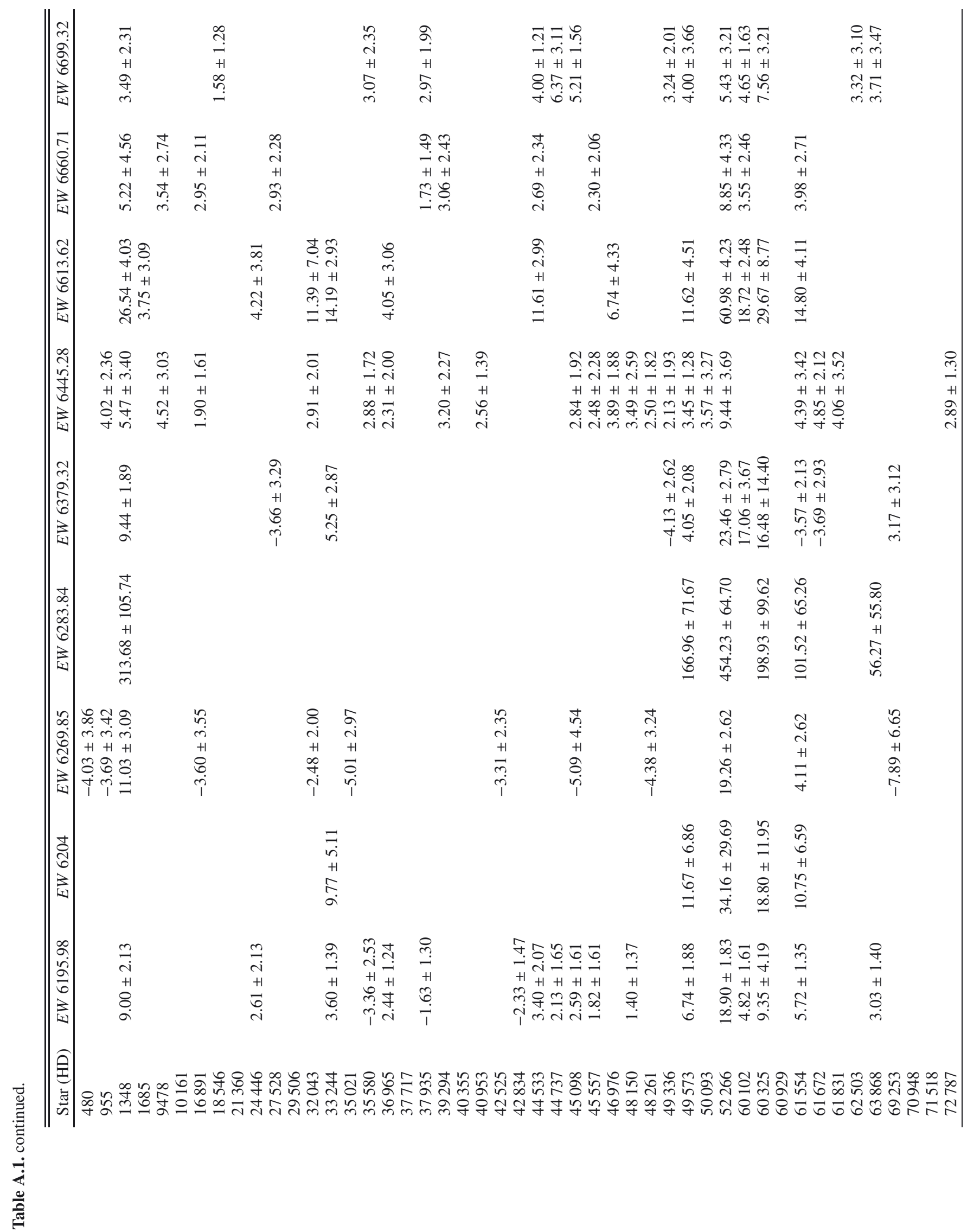




\begin{tabular}{|c|c|c|c|c|c|c|c|c|c|c|c|c|c|c|c|c|c|c|}
\hline $\begin{array}{l}\text { cे } \\
8 \\
8 \\
0 \\
2\end{array}$ & & 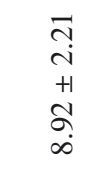 & & 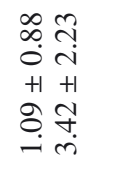 & $\begin{array}{l}\vec{\infty} \\
\stackrel{+}{+1} \\
\vec{\sigma} \\
-\end{array}$ & & & & 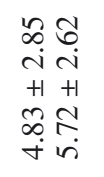 & 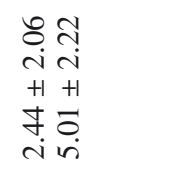 & & & $\begin{array}{ll}0 & 0 \\
+ & i \\
-i & i \\
+1 & +1 \\
0 & \stackrel{1}{i} \\
i & m\end{array}$ & & & $\begin{array}{l}0 \\
i n \\
i \\
+1 \\
+ \\
\infty \\
i\end{array}$ & $\begin{array}{l}i \\
i n \\
+1 \\
+ \\
\dot{d} \\
\dot{m}\end{array}$ & $\begin{array}{l}\delta \\
i \\
+1 \\
\vdots \\
\sigma\end{array}$ \\
\hline 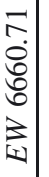 & & $\begin{array}{l}\text { ñ } \\
\text { bे } \\
+1 \\
\stackrel{2}{2} \\
\infty\end{array}$ & & & & & & & 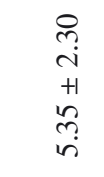 & $\begin{array}{l}n \\
\dot{c} \\
\dot{m} \\
+1 \\
\hat{m} \\
\dot{m}\end{array}$ & 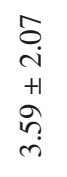 & & 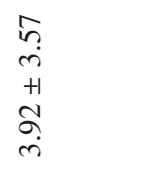 & & & 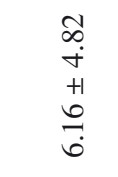 & & \\
\hline & 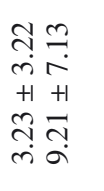 & $\begin{array}{l}\overrightarrow{7} \\
\dot{+} \\
+1 \\
6 \\
\dot{m} \\
\dot{m}\end{array}$ & & 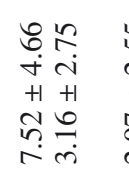 & 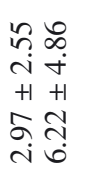 & 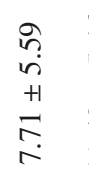 & $\begin{array}{l}o \\
+ \\
i n \\
+1 \\
+1 \\
\stackrel{+}{+} \\
=\end{array}$ & & 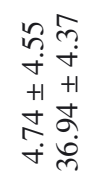 & 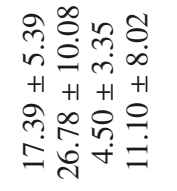 & $\begin{array}{l}7 . \\
0 \\
0 \\
+1 \\
+1 \\
0 \\
0 \\
0 \\
0.7\end{array}$ & 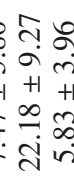 & 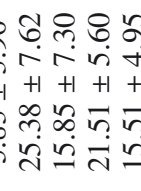 & 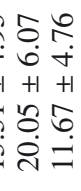 & 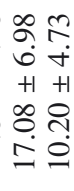 & 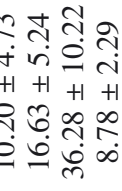 & 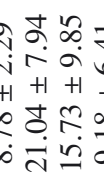 & 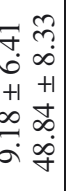 \\
\hline 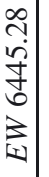 & 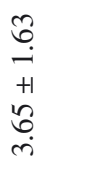 & 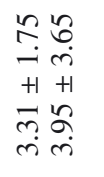 & $\begin{array}{l}\stackrel{n}{\dot{r}} \\
+1 \\
\stackrel{+1}{n}\end{array}$ & 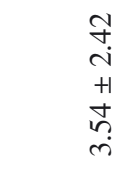 & & 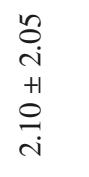 & & 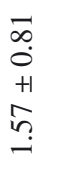 & 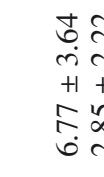 & 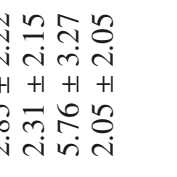 & & & 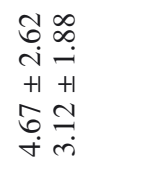 & 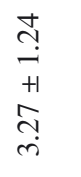 & $\begin{array}{l}\stackrel{2}{a} \\
i \\
+1 \\
0 \\
\stackrel{r}{r}\end{array}$ & 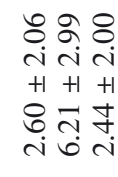 & & 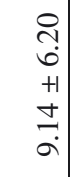 \\
\hline 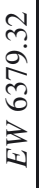 & & 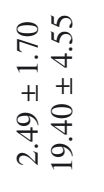 & & & & & & 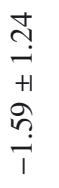 & $\begin{array}{l}+ \\
\infty \\
\dot{m} \\
+1 \\
0 \\
\stackrel{0}{\leq} \\
\end{array}$ & $\begin{array}{l}8 \\
i \\
+1 \\
\infty \\
\infty \\
\infty\end{array}$ & 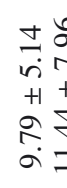 & & 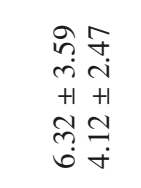 & \begin{tabular}{l}
$\stackrel{n}{a}$ \\
$\stackrel{+}{+1}$ \\
\multirow{2}{+}{} \\
$\stackrel{+}{+}$
\end{tabular} & 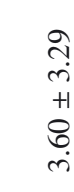 & 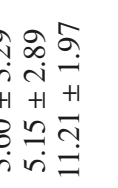 & & 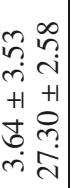 \\
\hline 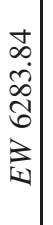 & & $\begin{array}{l}30 \\
\dot{\sigma} \\
+1 \\
+ \\
\hat{\sigma} \\
\dot{d}\end{array}$ & & & $\begin{array}{l}+ \\
\dot{0} \\
0 \\
+ \\
+1 \\
+1 \\
0 \\
\dot{J} \\
=\end{array}$ & $\begin{array}{l}\stackrel{\circ}{i} \\
\dot{\infty} \\
+1 \\
o \\
\stackrel{0}{i} \\
\infty\end{array}$ & $\begin{array}{l}a \\
\stackrel{2}{2} \\
+1 \\
m \\
0 \\
0\end{array}$ & & 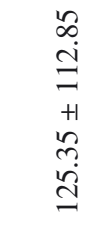 & 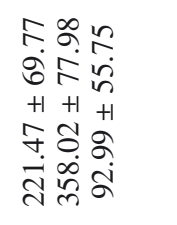 & 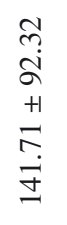 & 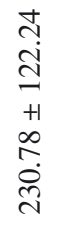 & 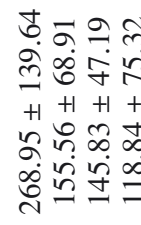 & 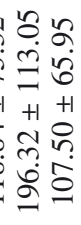 & 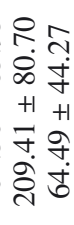 & 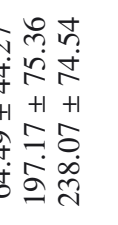 & 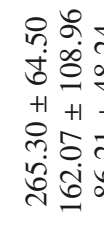 & 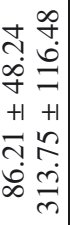 \\
\hline $\begin{array}{l}\infty \\
0 \\
0 \\
0 \\
0 \\
>\end{array}$ & & 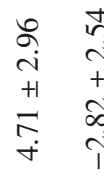 & & $\begin{array}{l}n \\
\infty \\
\infty \\
+1 \\
0 \\
\stackrel{1}{1} \\
= \\
1\end{array}$ & 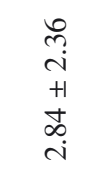 & & & & 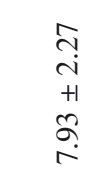 & $\begin{array}{l}\stackrel{\infty}{+} \\
\stackrel{+}{+} \\
+ \\
\vec{\sigma} \\
\dot{\sigma}\end{array}$ & & $\begin{array}{l}0 \\
\dot{m} \\
+1 \\
\infty \\
\infty \\
0 \\
0\end{array}$ & 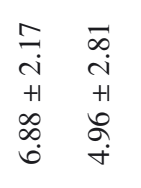 & 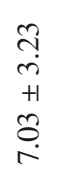 & & 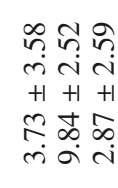 & 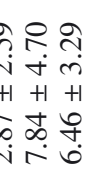 & $\begin{array}{l}\triangleright \\
\infty \\
\dot{r} \\
+1 \\
2 \\
\dot{ \pm} \\
\dot{ \pm}\end{array}$ \\
\hline $\begin{array}{l}\text { d } \\
\text { d } \\
\text { ते }\end{array}$ & & 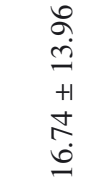 & & & & & $\begin{array}{l}\infty \\
\vdots \\
0 \\
+1 \\
\overrightarrow{+} \\
\stackrel{2}{2}\end{array}$ & & 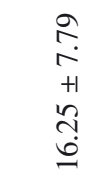 & 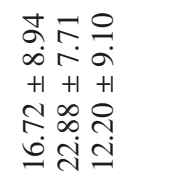 & & $\begin{array}{l}\text { oे } \\
0 \\
+1 \\
+\infty \\
0 \\
0 \\
0\end{array}$ & 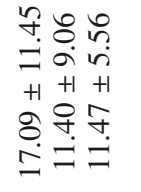 & 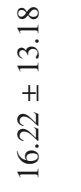 & 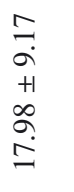 & 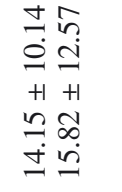 & 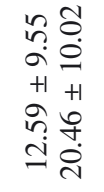 & 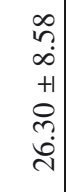 \\
\hline $\begin{array}{l}\infty \\
\vdots \\
\vdots \\
0 \\
3\end{array}$ & 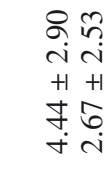 & 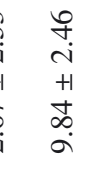 & $\begin{array}{l}\vec{b} \\
\stackrel{+}{+1} \\
\stackrel{n}{a} \\
\stackrel{-}{-}\end{array}$ & 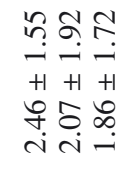 & 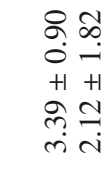 & 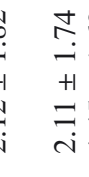 & 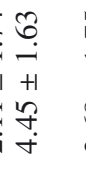 & \begin{tabular}{l}
$n$ \\
\multirow{2}{*}{} \\
+1 \\
$\infty$ \\
0 \\
$i$ \\
$i$
\end{tabular} & $\begin{array}{l}a \\
a \\
i \\
+1 \\
o \\
\stackrel{r}{r}\end{array}$ & 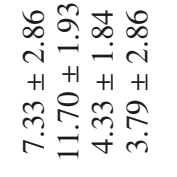 & $\begin{array}{l}\hat{n} \\
\text { i } \\
+1 \\
o \\
0 \\
0\end{array}$ & 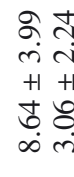 & 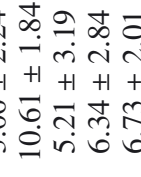 & 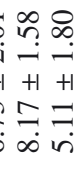 & 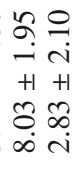 & 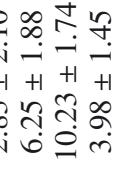 & 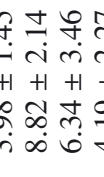 & 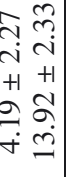 \\
\hline & 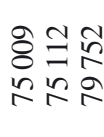 & $10 \mathrm{~m}$ & & & 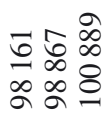 & $\begin{array}{l}0 \\
0 \\
0 \\
0 \\
8\end{array}$ & s. & 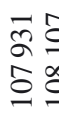 & $\begin{array}{l}\hat{0} \\
\text { id } \\
\infty \\
\infty\end{array}$ & $\begin{array}{l}\hat{2} \\
\text { aे } \\
\text { a }\end{array}$ & 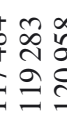 & & 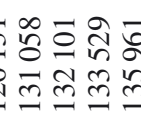 & बें̊ㅁ & 叧 & 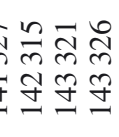 & 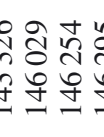 & \\
\hline
\end{tabular}




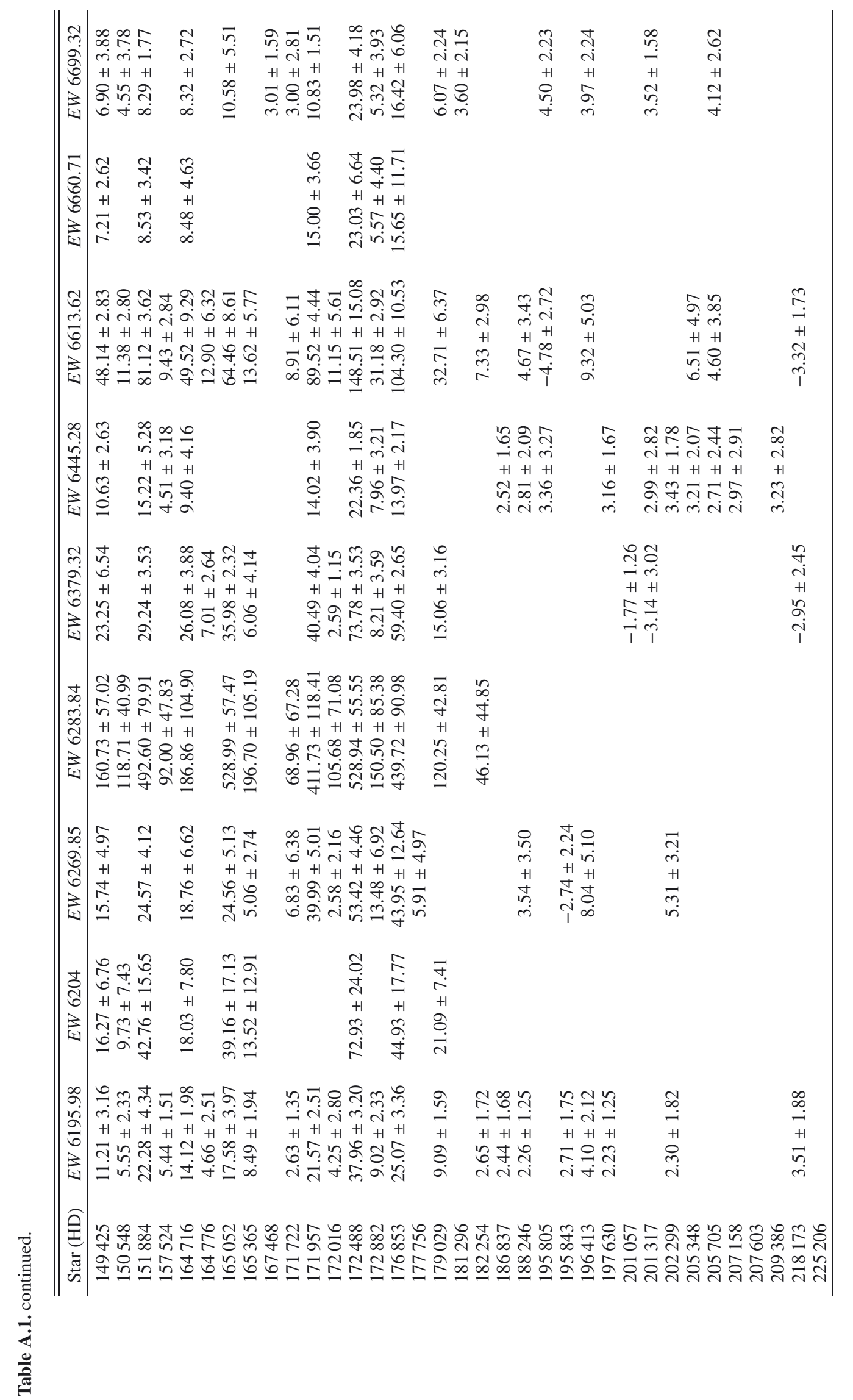

